\title{
A characterization of representation-finite algebras
}

\author{
by
}

Andrzej Skowroński and Michał Wenderlich (Toruń)

\begin{abstract}
Let $A$ be a finite-dimensional, basic, connected algebra over an algebraically closed field. Denote by $\Gamma(A)$ the Auslander-Reiten quiver of $A$. We show that $A$ is representation-finite if and only if $\Gamma(A)$ has at most finitely many vertices lying on oriented cycles and finitely many orbits with respect to the action of the Auslander-Reiten translation.
\end{abstract}

Let $K$ denote a fixed algebraically closed field and $A$ a finite-dimensional $K$-algebra (associative, with an identity) which we shall assume to be basic and connected. By an A-module is meant a finite-dimensional right $A$-module. Throughout the paper we shall freely use the terminology and notation introduced in [7]. In particular, we denote by $\bmod A$ the category of $A$-modules, by $\operatorname{rad}(\bmod A)$ the Jacobson radical of $\bmod A$, and by $\operatorname{rad}^{\infty}(\bmod A)$ the intersection of all powers $\operatorname{rad}^{i}(\bmod A), i \geq 0$, of $\operatorname{rad}(\bmod A)$. From the existence of the Auslander-Reiten sequences in $\bmod A$ we know that $\operatorname{rad}(\bmod A)$ is generated by irreducible maps as a left and as a right ideal. By $\Gamma(A)$ we denote the Auslander-Reiten quiver of $A$ whose vertices are the isoclasses of indecomposable objects in $\bmod A$ and arrows correspond to irreducible maps, and by $\tau$ and $\tau^{-1}$ the Auslander-Reiten translations $D \operatorname{Tr}$ and $\operatorname{Tr} D$, respectively. For the sake of simplicity we identify an $A$-module with its isomorphism class. The $\tau$-orbit of an indecomposable $A$-module $X$ is the family of non-zero modules of the form $\tau^{n} X$, $n \in \mathcal{Z}$, where $\mathcal{Z}$ is the set of all integers. An $A$-module $X$ is called periodic if $\tau^{n} X \simeq X$ for some $n \neq 0$. By a path from $M$ to $N$ in $\Gamma(A)$ we mean a sequence of vertices and arrows $M \rightarrow M_{1} \rightarrow \ldots \rightarrow M_{n} \rightarrow N$ in $\Gamma(A)$. In this case, $M$ is called a predecessor of $N$ and $N$ a successor of $M$. An oriented cycle is a non-trivial path from a point to itself. Recall that an algebra $A$ is called representation-finite if $\Gamma(A)$ is finite. In [4] the following results were proved:

(a) An algebra $A$ is representation-finite if and only if $\operatorname{rad}^{\infty}(\bmod A)=0$. 
(b) If $\operatorname{rad}^{\infty}(\bmod A)$ is nilpotent, then $A$ is tame (in the sense of [1] and $[8])$.

(c) If $A$ is either a tilted algebra or a standard selfinjective algebra then $\operatorname{rad}^{\infty}(\bmod A)$ is nilpotent if and only if $A$ is domestic (in the sense of [8]).

Representation-finite algebras are domestic and domestic algebras are tame (see [8]).

We shall prove here the following characterization of representation-finite algebras.

Theorem. Let $A$ be an algebra. The following conditions are equivalent.

(i) $A$ is representation-finite.

(ii) $\Gamma(A)$ admits at most finitely many vertices lying on an oriented cycle and the number of $\tau$-orbits in $\Gamma(A)$ is finite. finite.

(iii) $\operatorname{rad}^{\infty}(\bmod A)$ is nilpotent and the number of $\tau$-orbits in $\Gamma(A)$ is

Proof. Obviously (i) implies (ii). Moreover, (iii) implies (i). Indeed, suppose that $A$ is representation-infinite and satisfies (iii). Then the nilpotency of $\operatorname{rad}^{\infty}(\bmod A)$ implies that $A$ is tame and then, by the validity of the Brauer-Thrall II conjecture (for a proof, see for example [2]) and $[1,6.7]$, there are infinitely many pairwise non-isomorphic indecomposable $A$-modules $X$ with $X \simeq \tau X$, impossible by the second part of (iii).

Therefore, in order to prove the theorem, it is enough to show that (ii) implies the nilpotency of $\operatorname{rad}^{\infty}(\bmod A)$.

Observe first that, if $M_{0} \rightarrow M_{1} \rightarrow \ldots \rightarrow M_{n} \rightarrow M_{0}$ is an oriented cycle in $\Gamma(A)$, then either all modules $M_{i}$ are periodic or $\tau^{m} M_{i}=0$ (resp. $\left.\tau^{-m} M_{i}=0\right)$ for some $m>0$ and some $i$. Indeed, if one of the modules $M_{i}$, say $M_{0}$, is not periodic and $\tau^{m} M_{i} \neq 0$ for all $m \geq 0$ (resp. $m \leq 0$ ) and all $i, 0 \leq i \leq n$, then the modules $\tau^{m} M_{0}, m \geq 0$ (resp. $m \leq 0$ ) are pairwise non-isomorphic and lie on oriented cycles $\tau^{m} M_{0} \rightarrow \tau^{m} M_{1} \rightarrow \ldots \rightarrow$ $\tau^{m} M_{n} \rightarrow \tau^{m} M_{0}$, a contradiction to (ii).

Denote by $\Gamma^{+}(A)$ (resp. $\left.\Gamma^{-}(A)\right)$ the full translation subquiver of $\Gamma(A)$ formed by all non-periodic indecomposable modules $X$ such that $\tau^{n} X \neq 0$ (resp. $\tau^{-n} X \neq 0$ ) for all $n \geq 0$. By the above remark, $\Gamma^{+}(A)$ and $\Gamma^{-}(A)$ are quivers without oriented cycles. Then there exist finite sets $\mathcal{X}$ and $\mathcal{Y}$ of indecomposable $A$-modules such that the following conditions are satisfied:

(1) $\mathcal{X}$ (resp. $\mathcal{Y})$ intersects every $\tau$-orbit in $\Gamma^{+}(A)$ (resp. $\left.\Gamma^{-}(A)\right)$.

(2) Every path in $\Gamma(A)$ with source and target in $\mathcal{X}$ (resp. $\mathcal{Y}$ ) has all vertices in $\mathcal{X}$ (resp. $\mathcal{Y})$.

(3) There is no oriented cycle in $\Gamma(A)$ consisting of modules from $\mathcal{X}$ (resp. $\mathcal{Y})$. 
(4) Every predecessor (resp. successor) of some module of $\mathcal{X}$ (resp. $\mathcal{Y}$ ) belongs to $\Gamma^{+}(A)$ (resp. $\left.\Gamma^{-}(A)\right)$.

Denote by $\mathcal{C}^{+}\left(\right.$resp. $\left.\mathcal{C}^{-}\right)$the full translation subquiver of $\Gamma(A)$ formed by all proper predecessors (resp. proper successors) of modules of $\mathcal{X}$ (resp. $\mathcal{Y}$ ). We may assume that $\mathcal{C}^{+}$and $\mathcal{C}^{-}$are disjoint. Observe that $\mathcal{C}^{+}$(resp. $\mathcal{C}^{-}$) is a disjoint union of translation quivers of the form $(-N) \Delta$ (resp. $N \Delta$ ) for some quiver $\Delta$ without oriented cycles. Let $\mathcal{D}$ be the family of all indecomposable $A$-modules which are neither in $\mathcal{C}^{+}$nor in $\mathcal{C}^{-}$. Then $\mathcal{D}$ is (up to isomorphism) finite; denote by $d$ the maximum of dimensions of modules from $\mathcal{D}$.

Now let $M$ and $N$ be two indecomposable $A$-modules and $f: M \rightarrow N$ a non-zero map in $\operatorname{rad}^{\infty}(\bmod A)$. Assume that $M$ does not belong to $\mathcal{C}^{-}$. We claim that $f$ factors through a direct sum of modules of $\mathcal{Y}$. Since $f \in$ $\operatorname{rad}^{\infty}(M, N)$, there exists, for each $t>0$, a chain

$$
M \stackrel{g_{1}}{\rightarrow} M_{1} \stackrel{g_{2}}{\rightarrow} M_{2} \rightarrow \ldots \rightarrow M_{t-1} \stackrel{g_{t}}{\rightarrow} M_{t}
$$

of irreducible maps in $\bmod A$ and a morphism $h_{t} \in \operatorname{rad}^{\infty}\left(M_{t}, N\right)$ such that $f=h_{t} g_{t} \ldots g_{1}$. Then there exists $p \geq 0$ such that, for $t \geq p, M_{t}$ does not contain direct summands from $\mathcal{C}^{+}$. Applying the lemma of Harada and Sai [3] (for a proof we refer to [6, 2.2]) we conclude that, for $t \geq p+2^{d}, M_{t}$ is a direct sum of modules of $\mathcal{C}^{-}$. Observe that, for $s=p+2^{d}, g_{s} \ldots g_{1}$ is a linear combination of paths in $\Gamma(A)$ from $M$ to indecomposable direct summands of $M_{s}$, hence lying in $\mathcal{C}^{-}$, which must factor through modules of $\mathcal{Y}$. Similarly, we show that, if $N$ is not in $\mathcal{C}^{+}$, then $f$ factors through a direct sum of modules of $\mathcal{X}$. Let now $m=2^{d}+1$. We shall show that $\left(\operatorname{rad}^{\infty}(\bmod A)\right)^{2 m}=$ 0 . It is enough to show that for each chain of morphisms

$$
Z_{0} \stackrel{u_{1}}{\rightarrow} Z_{1} \stackrel{u_{2}}{\rightarrow} Z_{2} \rightarrow \ldots \rightarrow Z_{2 m-1} \stackrel{u_{2 m}}{\rightarrow} Z_{2 m}
$$

where all $Z_{i}$ are indecomposable $A$-modules and where the $u_{i}$ belong to $\operatorname{rad}^{\infty}(\bmod A)$, the composition $u=u_{2 m} \ldots u_{1}$ is zero. Since we assume that $\mathcal{C}^{+}$and $\mathcal{C}^{-}$are disjoint, it follows that either $u_{i}$ factors through a direct sum of modules of $\mathcal{X}$ or $u_{i+1}$ factors through a direct sum of modules of $\mathcal{Y}$. Consequently, for each $j, 1 \leq j \leq m$, we have $u_{2 j} u_{2 j-1}=\beta_{j} \alpha_{j}$, where $\alpha_{j} \in$ $\operatorname{rad}\left(Z_{2 j-2}, V_{j}\right), \beta_{j} \in \operatorname{rad}\left(V_{j}, Z_{2 j}\right)$ and $V_{j}$ is a direct sum of indecomposable modules of $\mathcal{X} \cup \mathcal{Y}$. Let $\gamma_{j}=\alpha_{j+1} \beta_{j}$ for $j=1, \ldots, m-1$. Applying again the lemma of Harada and Sai, we conclude that $\gamma_{m-1} \ldots \gamma_{1}=0$. Hence $u=\beta_{m} \gamma_{m-1} \cdots \gamma_{1} \alpha_{1}=0$, which finishes the proof of the theorem.

The following corollary is an immediate consequence of the theorem.

COROllary 1. Let $A$ be an algebra such that $\Gamma(A)$ has no oriented cycles. Then $A$ is representation-finite if and only if the number of $\tau$-orbits in $\Gamma(A)$ is finite. 
Observe that wild hereditary algebras are representation-infinite and their Auslander-Reiten quiver does not contain oriented cycles (see [5]). Recall also that an algebra $A$ is called representation-directed provided every indecomposable $A$-module is directing, that is, it does not belong to an oriented cycle $M_{0} \rightarrow M_{1} \rightarrow \ldots \rightarrow M_{n} \rightarrow M_{0}$ of non-zero non-isomorphisms between indecomposable $A$-modules $M_{i}$. It was recently shown in [9] that a connected Auslander-Reiten component consisting of directing modules has only finitely many $\tau$-orbits and the number of such components is finite. Hence the above corollary also implies the following characterization of representation-directed algebras due to Ringel (see [7, 2.4]).

Corollary 2. An algebra $A$ is representation-directed if and only if $A$ is representation-finite and $\Gamma(A)$ has no oriented cycles.

The authors wish to thank the Polish Ministry of Education for its support under Research Project R.P.I.10.

\section{References}

[1] W. W. Crawley-Boevey, On tame algebras and bocses, Proc. London Math. Soc. 56 (1988), 451-483.

[2] U. Fischbacher, Une nouvelle preuve d'un théorème de Nazarova et Roiter, C. R. Acad. Sci. Paris Sér. I 300 (9) (1985), 259-262.

[3] M. Har ad a and Y. Sai, On categories of indecomposable modules I, Osaka J. Math. 7 (1970), 323-344.

[4] O. Kerner and A. Skowroński, On module categories with nilpotent infinite radical, Compositio Math. 77 (1991), 313-333.

[5] C. M. Ringel, Finite-dimensional hereditary algebras of wild representation type, Math. Z. 161 (1978), 235-255.

[6] -, Report on the Brauer-Thrall conjectures, in: Proc. ICRA II (Ottawa 1979), Lecture Notes in Math. 831, Springer, 1980, 104-136.

[7] - , Tame Algebras and Integral Quadratic Forms, Lecture Notes in Math. 1099, Springer, 1984

[8] A. Skowroński, Algebras of polynomial growth, in: Topics in Algebra, Banach Center Publ. 26, Part 1, PWN, Warszawa 1990, 535-568.

[9] A. Skowroński and S. O. Smalø, Directing modules, J. Algebra, to appear.

INSTITUTE OF MATHEMATICS

NICHOLAS COPERNICUS UNIVERSITY

CHOPINA $12 / 18$

87-100 TORUŃ, POLAND 